\title{
Non-linear Control Law for Articulated Serial Manipulators: Simulation Augmented with Hardware Implementation
}

\author{
Muhammad Imran Ullah ${ }^{1}$, Syed Ali Ajwad ${ }^{1}$, Muhammad Irfan ${ }^{2}$, Jamshed Iqbal ${ }^{1,3}$ \\ ${ }^{I}$ Department of Electrical Engineering, COMSATS Institute of Information Technology, \\ Islamabad, Pakistan \\ ${ }^{2}$ Department of Electrical Engineering, University of Engineering and Technology, \\ Taxila, Pakistan \\ ${ }^{3}$ Department of Electrical Engineering, National University of Computer and Emerging Sciences, \\ Islamabad, Pakistan \\ jamshed.iqbal@nu.edu.pk
}

\begin{abstract}
The performance of a robotic arm includes accuracy, repeatability and reliability to accomplish a task. These parameters, in turn, are function of associated control law. Multi-Degree Of Freedom (DOF) robotic arms, because of their inherent highly non-linear dynamics, demand sophisticated control laws. Trivial control strategies fail to cope with disturbances and uncertainties that are common in today's plants. This paper presents the design, simulation and physical implementation of a non-linear control technique Variable Structure Control (VSC) for a 6 DOF arm. Based on the derived dynamic model of the arm and designed control law, simulations have been conducted in MATLAB/Simulink. The controller parameters have been tuned for optimal response. Various desired trajectories characterize the tracking performance of the control law. The simulation results have been then validated by implementing the law on a customdeveloped novel AUTonomous Articulated Robotic Educational Platform (AUTAREP). Coupling effects between various joints of the robot have also been investigated. Results of this research find potential in industrial control of robotic manipulators to perform complex tasks.
\end{abstract}

Index Terms-Arm manipulator, robot control, robust control, variable structure control.

\section{INTRODUCTION}

Robots are machines developed to perform a task automatically or by following the instructions through remote control. Robots are being used for various applications including rehabilitation [1], [2], assistance [3], [4], Virtual Reality (VR) [5], rescue [6] and in other domains like industrial [7], space [8], nuclear power plants [9], underwater [10] and so on. The scope of the present paper is related with industrial sector where sophisticated scheme is required to control the robots [11] so as to enhance accuracy, repeatability and reliability [12]. The main challenge in the motion control of rigid manipulators is the complexity of their dynamics and uncertainties, which result from nonlinearities and coupling in the robotic manipulators. Based on this knowledge, controller design becomes a challenging task.

Manuscript received 28 December, 2014; accepted 29 May, 2015.
Scientific literature reports various linear as well as nonlinear control strategies for multi-Degree Of Freedom (DOF) robotic manipulators. The most popular linear control technique is based on Proportional-IntegralDerivative (PID) of the error signal [13]. A PID controller for 3 DOF serial link exoskeleton robot based on minimum jerk trajectory algorithm is implemented by Iqbal et al. [14], [15]. Antonio et al. [16] have proposed PID control design with bounded torque conditions satisfying asymptotic stability criteria. Conventional control algorithms like PID have limitations and drawbacks. There is no particular method for tuning and adjustment of the associated gains. PID controllers are effective for linear systems but not suitable for nonlinear and complex systems. With simple PID controller and Linear Quadratic Regulator (LQR), it is very difficult to control multi-DOF non-linear robotic arm manipulator. The classical control design techniques can also be combined with modern techniques to give better improved response for uncertain systems and can address chattering and gain adjustment issues [17]. Farzin et al. [18] and Imran et al. [19] have proposed control techniques combining Proportional-Derivative PD and PID control with Computed Torque Control (CTC). Jingmei et al. [20] have developed an approach for improving performance of the robotic arm by combining PID, adaptive fast method and Variable Structure Control (VSC) approach. Dimitri et al. [21] have designed a VSC scheme to overcome chattering phenomenon by using adaptive gains. Jing et al. [22] have presented VSC approach using integral sliding surface and adaptive switching for estimating uncertain system parameters.

The aforementioned and most of the reported literature on modern control techniques for multi-DOF robotic arms is limited to software simulations. The proposed work presents details for software as well as hardware implementation of VSC control law for a 6 DOF arm.

The paper is organized as follows: Section II briefly introduces custom-developed manipulator for the present study. Section III discusses VSC control. In Section IV, the simulation analysis of VSC is carried out. Hardware 
implementation for the control strategy is covered in Section V. Finally, Section VI comments on conclusions.

\section{ROBOTIC ARM MANIPULATOR}

An AUTonomous Articulated Robotic Educational Platform (AUTAREP) [23] has been developed for physical realization of the control strategy. The platform is centred on a 6 DOF serial manipulator with all joints as revolute. The arm's geometrical configuration is made up of waist, shoulder, elbow and wrist. The arm is fully actuated by six precise DC servo motors with wrist actuated with 2 DOF. The platform is illustrated in Fig. 1.

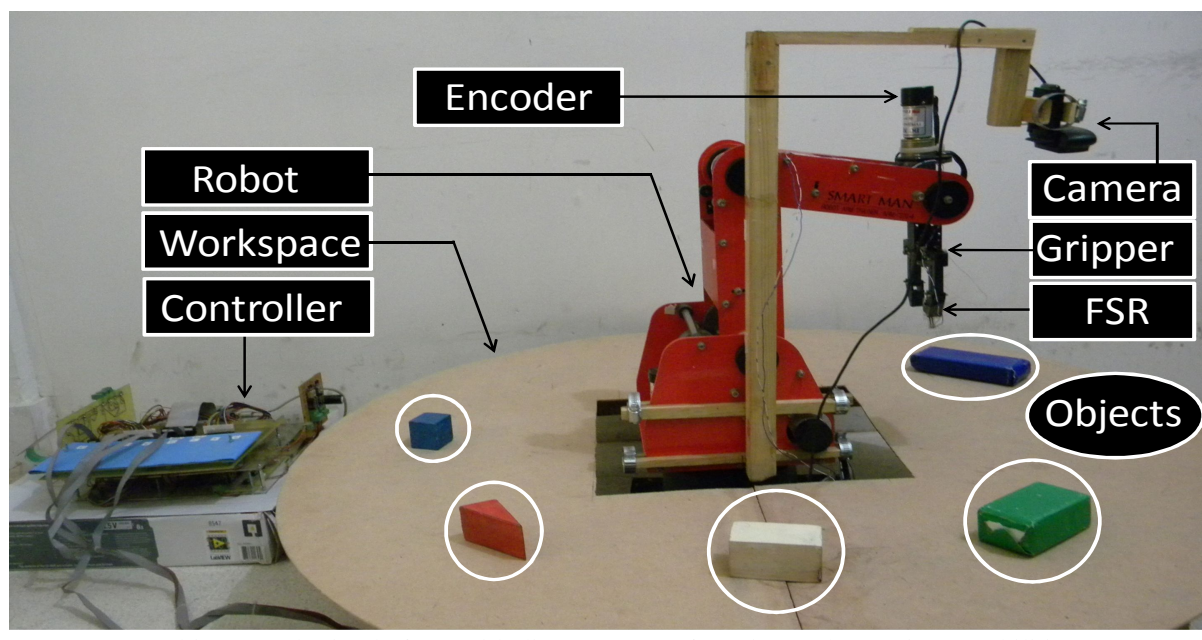

Fig. 1. UTAREP - An open source multi-DOF robotic platform with diverse range of sensing and actuation capabilities [23].

\section{VARIABLE STRUCTURE CONTROL}

VSC is a robust non-linear control technique, which is used to eliminate disturbances and uncertainties. Its main feature is to drive the system states to a switching surface. Then the system operates in the sliding mode to minimize the modelling uncertainties and disturbances [24]. The dynamics of a nonlinear system alters with system's states which are estimated or available through feedback. Generally a high-frequency switching control is utilized to ensure less sensitivity towards uncertainties in the plant's parameters.

In VSC design, the most important step is switching control which drives the plant states to the selected switching surface and keeps it there for all future times. To characterize this step, a Lyapunov function is defined on state variables which must remain positive and becomes zero only when state variables are zero.

The condition under which derivative of a function is always negative also ensures that variables approach zero in finite time. Firstly, the sliding manifold is designed as

$$
S=C e+\dot{e},
$$

where $C$ is sliding surface constant that must satisfy Hurwitz condition, i.e. $C>0$ and $\dot{e}$ is the derivative of the error signal vector defined as the difference between the present and desired joint trajectory i.e. $e=q-q_{d}$. For simplicity, $C$ is taken as a positive definite diagonal matrix i.e. $C=\operatorname{diag}\left\{c_{1} c_{2} c_{3} c_{4}\right\}$. Mathematically when $S=0$, (1) reduces to (2)

$$
\dot{e}=-C e \text {. }
$$

This is a first order differential equation which has the solution given by (3)

$$
e(t)=e(0) \exp (-C t)
$$

This shows that the error reduces to zero exponentially for each joint under the selected conditions.

VSC consists of two components which are equivalent control and robust control. The equivalent control part must ensure that the states of the closed-loop system follow the sliding manifold surface to the dynamic equilibrium of the error dynamic system. To calculate the equivalent control, differentiating (1) w.r.t. time and setting $S=0$ result in (4)

$$
0=C \dot{e}+\ddot{e}
$$

The general dynamic model equation for $\mathrm{n}$-serial link robotic arm is given by (5)

$$
\ddot{q}=M^{-1}\{\tau-V(q, \dot{q})-G(q)\},
$$

where $M(q)$ is a $n \times n$ inertia matrix, $V(q, \dot{q})$ and $G(q)$ are $n \times 1$ vectors of Coriolis centrifugal force, Gravitational force and Frictional force vectors respectively. $\tau$ is the $n \times 1$ torque vector applied to the joints of the robot and $q, \dot{q}$ and $\ddot{q}$ are $n \times 1$ vectors for angular position, velocity and acceleration respectively. Rearranging (5) gives the equivalent control part as expressed in (6)

$$
\tau_{e q}=M\left(\ddot{q}_{d}-C \dot{e}\right)+V+G .
$$

The equivalent control is designed with the assumption that the system's states are on the surface. VSC design involves the reaching phase (i.e. time required by states to reach at the sliding surface), sliding phase (i.e. time required by states to reach at the states equilibrium) and the sliding surfaces. To make this assumption true and to compensate for the dynamic model uncertainties, a discontinuous function of sliding manifold $S$ is required. For simplicity, we take

$$
\tau_{\text {disc. }}=-K \operatorname{sgn}(S)=-K \operatorname{sgn}(C e+\dot{e}),
$$


where $K$ is the discontinuity gain matrix having diagonal components i.e. $K=\operatorname{diag}\left\{k_{1} \mathrm{k}_{2} \mathrm{k}_{3} \mathrm{k}_{4}\right\}$. The complete control law can be written as in (8)

$$
\tau=M\left(\ddot{q}_{d}-C \dot{e}\right)+V+G-K \operatorname{sgn}(C e+\dot{e}) .
$$

To investigate restriction on $K$, a Lyapunov function $V$ is defined as (9):

$$
\begin{gathered}
V=0.5 S^{2}, \\
\dot{V}=S \dot{S} .
\end{gathered}
$$

Using (9) in (5) and rearranging results, (11) is obtained, which always ensures the finite time regulation and stability of the system

$$
\dot{V}=S\{-K \operatorname{sgn}(s)\}=-K|S| .
$$

\section{Simulation Results}

Simulation model developed in MATLAB/Simulink mainly consists of two s-functions. The controller governing equation is represented with the first function ' $V S C_{-} c o n t$ ' while derived dynamic model of the plant in [23] is coded in the second function 'AUTAREP_plant'. Desired angle $\left(q_{d}\right)$ and its first and second order derivatives are generated in subsystem block 'Des_j_angles'.

The designed controller and modelled plant is subjected to various trajectories including step, ramp and sinusoidal. The effect of inter-related constants $\mathrm{C}$ and $\mathrm{K}$ on the plant output has been investigated and optimum values of these matrices have been selected based on the simulated responses. For example, in case of joint 1 (waist joint), keeping $\mathrm{k}_{1}$ as constant, the step response of the joint for different $c_{1}$ values is illustrated in Fig. 2. It can be inferred that setting $c_{1}=4$ gives optimum settling time.

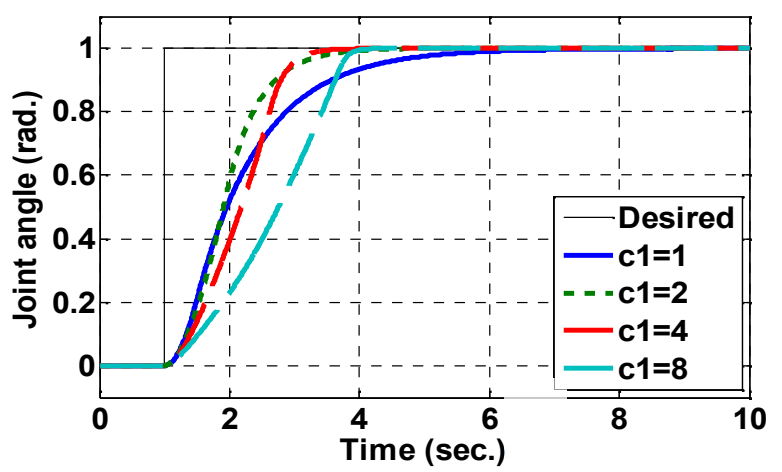

Fig. 2. Step response of waist joint for different $c_{1}$ values $\left(\mathrm{k}_{1}=2\right)$.

Tuning other joints in similar fashion resulted in the selected matrices as $\mathrm{C}=\operatorname{diag}\left\{\begin{array}{llll}4 & 16 & 4 & 1.5\end{array}\right\}$ and $\mathrm{K}=\operatorname{diag}\{22$ $10.2\}$. Corresponding step response of various joints moving simultaneously is shown in Fig. 3. It can be inferred from the plot that elbow joint and wrist joint even after reaching their desire position, are not stable till the shoulderjoint is reached at its destination and stabilized. This is in accordance with coupling effects of the joints.

Continuing with the tuned values of $C$ and $K$, the responses corresponding to ramp and sinusoidal inputs are illustrated in Fig. 4 and Fig. 5 respectively.

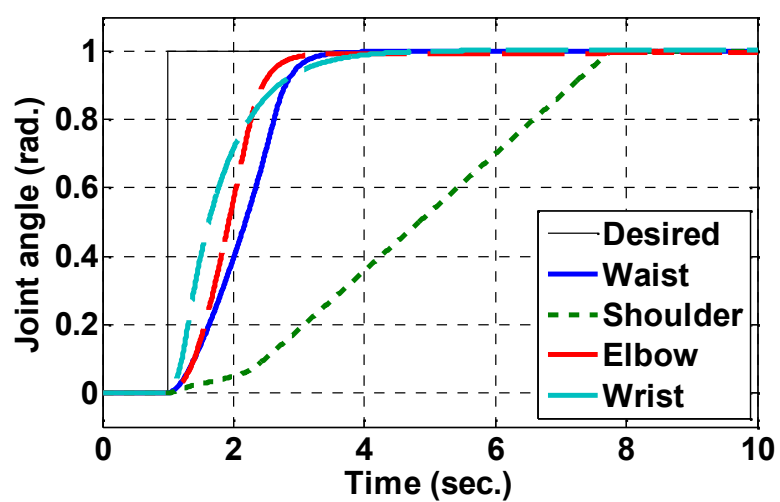

Fig. 3. Step response of various joints moving together.

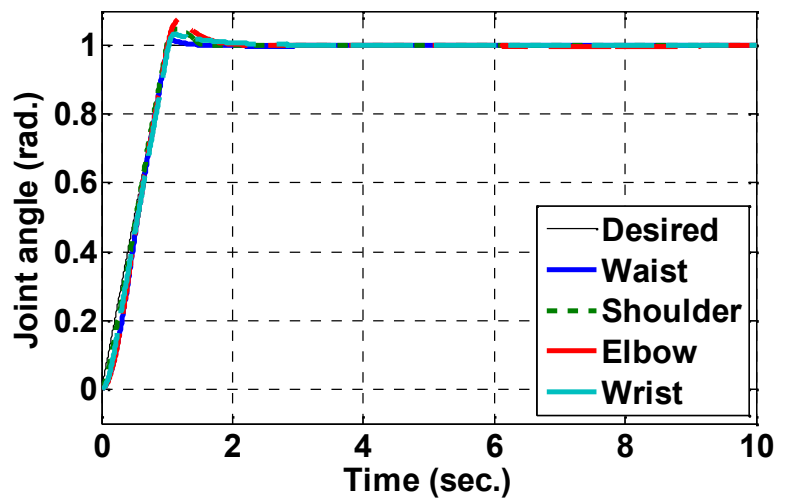

Fig. 4. Ramp response of various joints.

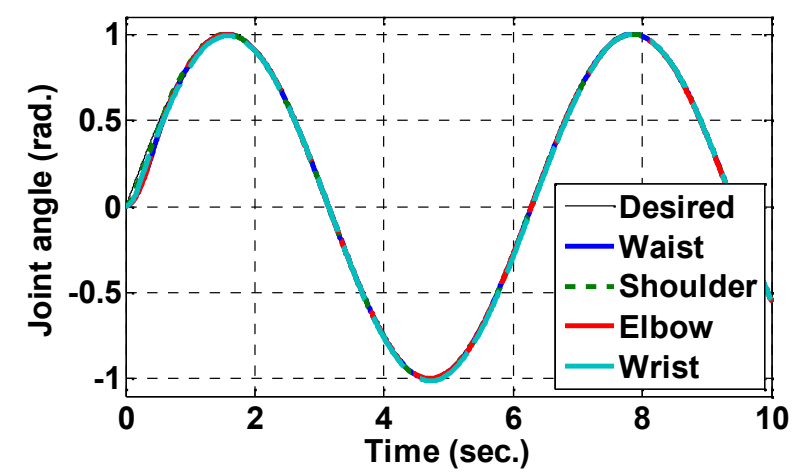

Fig. 5. Sinusoidal response of various joints.

All of the above simulation results confirm that VSC works well in simulation thus dictating that the developed VSC control law is good enough to be implemented on the real platform.

\section{HARDWARE IMPLEMENTATION}

The developed electronic hardware of AUTAREP, shown in Fig. 6, is centred on a 16-bit embedded controller dsPIC33F. Custom-designed motor drivers of rating 50 V/5 A actuate the platform's motors through Pulse Width Modulation (PWM) signals. The robotic arm can be controlled via a host PC as well as through a dedicated teaching pendent. The designed Graphic User Interface (GUI) permits a scientist to interact with the platform using intensive library containing more than 100 kernel commands. Details about the developed hardware and software are discussed in [25].

For implementation of VSC on hardware, in addition to simple trajectory tracking, the coupling effects have also been investigated. For this purpose, the experiment has been conducted in two different modes. 


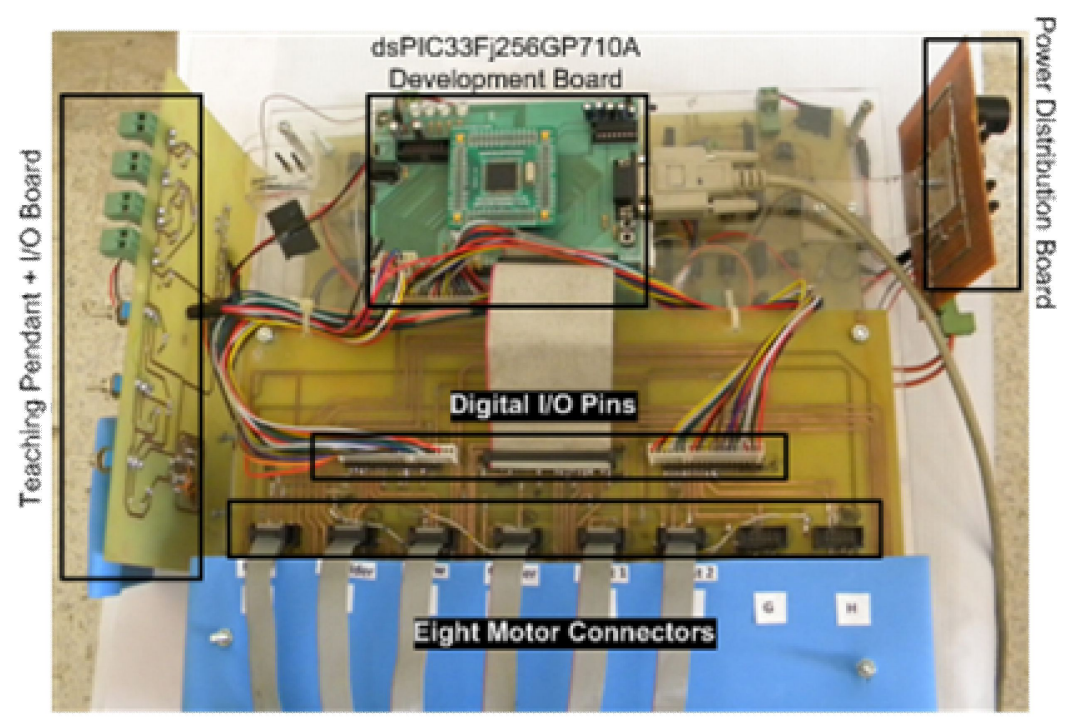

Fig. 6. The developed electronic hardware of AUTAREP.

In the first mode, the first three joints i.e. base, shoulder and elbow have been moved independently one after the other by 20 degree. The result of the joints' motion has been recorded and is plotted on the same graph illustrated in Fig. 7(a). In the second mode, all the joints have been moved simultaneously. The result of responses corresponding to the three joints in this case is plotted in Fig. 7(b), where again the desired joint angle movement is 20 degree for all joints.

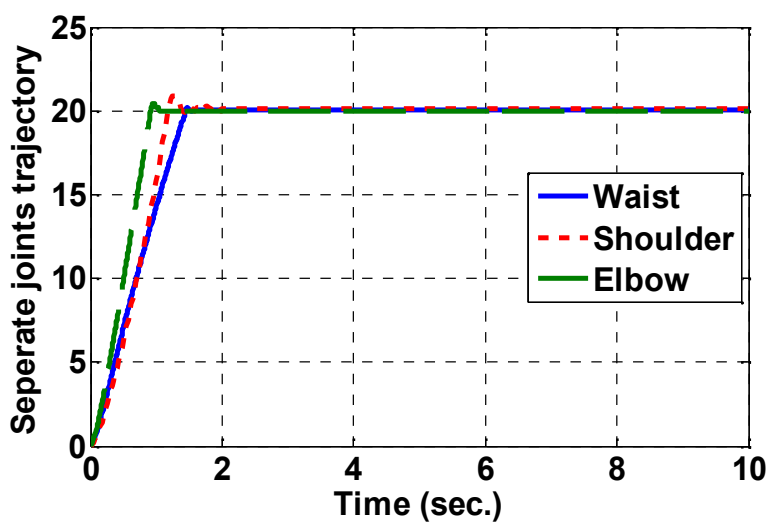

(a)

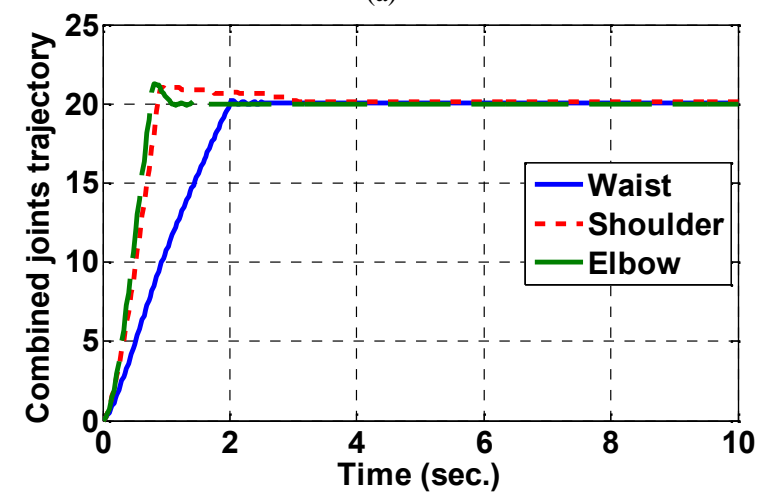

(b)

Fig. 7. Step-responses corresponding to first three joints actuated: (a) independently; (b) simultaneously.

Comparing results of both modes, it is observed that the overshoot in shoulder joint lasts for a longer time and it reaches to the desired position after the elbow joint has settled. The coupling effect on the shoulder-joint causes it to move faster but on the other hand increase its stabilization time.

The least effected joint is elbow. This also can be intuitively observed in arm dynamics. Elbow is the joint which is least influenced by the dynamics of base and shoulder joint. The base joint settling time has been increased which has no coupling effect in theory.

Investigation on the issue revealed that the effect is due to sharing the same power source. To confirm this, the experiment has been conducted again with shoulder and elbow movement and their responses have been found to be almost identical.

\section{Conclusions}

This paper presents design, simulation and implementation of a robust non-linear control technique VSC. In VSC simulation, it is observed that the sliding surface constant and switching gain constant play an important role particularly in the case when all joints move simultaneously. These constants are related with each other due to coupling effects in the joints. For VSC hardware implementation, results obtained are almost the same as observed in simulation. It is envisaged in near future to develop application specific strategies to further unleash the potential of VSC in elimination of uncertainties and disturbances. Also, extending the control implementation to include 2 DOF wrist is quite foreseen.

\section{REFERENCES}

[1] J. Iqbal, A. H. Khan, N. G. Tsagarakis, D. G. Caldwell, "A novel exoskeleton robotic system for hand rehabilitation - Conceptualization to prototyping", Biocybern. Biomed. Eng, vol. 34, no. 2, pp. 79-89, 2014. [Online]. Available: http://dx.doi.org/10.1016/j.bbe. 2014.01.003

[2] J. Iqbal, N. G. Tsagarakis, A. E. Fiorilla, D. G. Caldwell, "A portable rehabilitation device for the hand", in Proc. 32 ${ }^{\text {nd }}$ annual IEEE Int Conf. of Engineering in Medicine and Biology Society, Buenos Aires, Argentina, 2010, pp. 3694-3697. [Online]. Available: http://dx.doi.org/10.1109/iembs.2010.5627448

[3] J. Iqbal, N. G. Tsagarakis, D. G. Caldwell, "A multi-DOF robotic exoskeleton interface for hand motion assistance", in Proc. $33^{\text {rd }}$ annual IEEE Int. Conf. of Engineering in Medicine and Biology Society, Boston, US, 2011, pp. 1575-1678. [Online]. Available: http://dx.doi.org/10.1109/iembs.2011.6090458

[4] A. A. Khan, S. Riaz, J. Iqbal, "Surface estimation of a pedestrian walk for outdoor use of power wheelchair based robot", Life Sci. J. - Acta Zhengzhou University Overseas Edition, vol. 10, no. 3, pp. 1697 $1704,2013$. 
[5] J. Iqbal, N. G. Tsagarakis, D. G. Caldwell, "Design of a wearable direct-driven optimized hand exoskeleton device", in Proc. 4th Int. Conf. Advances in Computer-Human Interactions, France, 2011, pp. $142-146$.

[6] S. A. Ajwad, J. Iqbal, "Recent advances and applications of tethered robotic systems", Sci. Int. (Lahore), vol. 26, no. 5, 2014.

[7] R. U. Islam, J. Iqbal, S. Manzoor, A. Khalid, S. Khan, "An autonomous image-guided robotic system simulating industrial applications", in Proc. $7^{\text {th }}$ IEEE Int. Conf. on System of Systems Engineering, Genova, Italy, 2012, pp. 344-349. [Online]. Available: http://dx.doi.org/10.1109/sysose.2012.6384195

[8] J. Iqbal, M. R. Saad, A. M. Tahir, A. Malik, "State estimation technique for a planetary robotic rover", Rev. Fac. Ing - Univ. Ant., vol. 73, pp. 58-68, 2014.

[9] J. Iqbal, A. Tahir, R. U. Islam, R. U. Nabi, "Robotics for nuclear power plants - Challenges and future perspectives", in Proc. IEEE Int. Conf. on Applied Robotics for the Power Industry, Zurich, Switzerland, 2012, pp. 151-156. [Online]. Available: http://dx.doi.org/10.1109/carpi.2012.6473373

[10] A. M. Tahir, J. Iqbal, "Underwater robotic vehicles: Latest development trends and potential challenges", Sci. Int. (Lahore), vol. 26, no. 3, pp. 1111-1117, 2014.

[11] T. Cerlinca, S. G. Pentiuc, V. Vlad, "Real-time 3D hand gestures recognition for manipulation of industrial robots", Elektronika ir Elektrotechnika, vol. 19, no. 2, pp. 3-8, 2013. [Online]. Available: http://dx.doi.org/10.5755/j01.eee.19.2.1329

[12] A. Meddahi, K. Baizid, A. Yousnadj, J. Iqbal, "API based graphical simulation of robotized sites", in Proc. $14^{\text {th }}$ IASTED Int. Conf. on Robotics and Applications, Massachusetts, US, 2009, pp. 485-492.

[13] D. Levisauskas, T. Tekorius, "Investigation of P and PD controllers' performance in control systems with steady-state error compensation", Elektronika ir Elektrotechnika, vol. 5, no. 121, pp. 63-68, 2012. [Online]. Available: http://dx.doi.org/10.5755/ j01.eee.121.5.1297

[14] J. Iqbal, N. G. Tsagarakis, D. G. Caldwell, "Human hand compatible underactuated exoskeleton robotic system", IET Electron. Lett., vol. 50, no. 7, pp. 494-496, 2014. [Online]. Available: http://dx.doi.org/10.1049/el.2014.0508

[15] J. Iqbal, B. Khelifa, "Stroke rehabilitation using exoskeleton based robotic exercisers: Mini review", Biomed. Res. - India, vol. 26, no. 1, pp. 197-201, 2015.

[16] Y. Antonio, S. Victor, M. V. Javier, "Global asymptotic stability of the classical PID controller by considering saturation effects in industrial robots", Int. J. Adv. Robot. Syst., vol. 8, pp. 34-42, 2011.

[17] S. A. Ajwad, M. I. Ullah, B. Khelifa, J. Iqbal, "A comprehensive state-of-the-art on control of industrial articulated robots", J. Balk Tribol. Assoc., vol. 20, no. 4, pp. 499-521, 2014.

[18] P. Farzin, H. Y. Mohammad, S. Mohammad, M. Ebrahim, H. Ali, "PUMA-560 robot manipulator position computed torque control methods using Matlab/Simulink and their integration into graduate nonlinear control and Matlab courses", Int. J. Robot. Autom. vol. 3 , pp. 167-191, 2012.

[19] M. I. Ullah, S. A. Ajwad, R. U. Islam, U. Iqbal, J. Iqbal, "Modeling and computed torque control of a 6 Degree of Freedom robotic arm", in Proc. IEEE Int. Conf. on Robotics and Emerging Allied Technologies in Engineering, Islamabad, Pakistan, 2014, pp. 133138. [Online]. Available: http://dx.doi.org/10.1109/icreate.2014 6828353

[20] Z. Jingmei, H. Haiyang, K. Bo, "Studies of adaptive control methods based on VSC for trajectory tracking of robotic manipulators", in Proc. Int. Conf. on Robotics and Biomimetics, Guangzhou, China, 2012, pp. 429-434.

[21] M. Dimitri, S. Anwari, "Robust adaptive control for robotic manipulator based on chattering free variable structure system", in Proc. IEEE Int. Conf. on Electrical Engineering and Informatics, Selangor, Malaysia, 2009, pp. 247-252.

[22] Z. Jing, G. Xin, L. En, "An adaptive variable structure controller for robotic manipulators", in Proc. $6^{\text {th }}$ Int. Forum on Strategic Technology, Harbin, China, 2011, pp. 351-355. [Online]. Available: http://dx.doi.org/10.1109/ifost.2011.6021038

[23] S. Manzoor, R. U. Islam, A. Khalid, A. Samad, J. Iqbal, "An opensource multi-DOF articulated robotic educational platform for autonomous object manipulation", Robot. Cim-int Manuf., vol. 30, no. 3, pp. 351-362, 2014. [Online]. Available: http://dx.doi.org/10.1016/j.rcim.2013.11.003

[24] C. Edwards, S. Spurgeon, Sliding mode control: Theory and applications, CRC Press, 1998

[25] U. Iqbal, A. Samad, Z. Nissa, J. Iqbal, "Embedded control system for AUTAREP - A novel AUTonomous Articulated Robotic Educational Platform", Teh. Vjesn., vol. 21, no. 6, pp. 1255-1261, 2014. 